\title{
Independent control of harmonic amplitudes and phases via a time-domain digital coding metasurface
}

\author{
Jun Yan Dai (1) ${ }^{1,2}$, Jie Zhao ${ }^{1,2}$, Qiang Cheng ${ }^{1,2}$ and Tie Jun Cuil ${ }^{1,2}$
}

\begin{abstract}
Harmonic manipulations are important for applications such as wireless communications, radar detection and biological monitoring. A general approach to tailor the harmonics involves the use of additional amplifiers and phase shifters for the precise control of harmonic amplitudes and phases after the mixing process; however, this approach leads to issues of high cost and system integration. Metasurfaces composed of a periodic array of subwavelength resonators provide additional degrees of freedom to realize customized responses to incident light and highlight the possibility for nonlinear control by taking advantage of time-domain properties. Here, we designed and experimentally characterized a reflective time-domain digital coding metasurface, with independent control of the harmonic amplitude and phase. As the reflection coefficient is dynamically modulated in a predefined way, a large conversion rate is observed from the carrier signal to the harmonic components, with magnitudes and phases that can be accurately and separately engineered. In addition, by encoding the reflection phases of the meta-atoms, beam scanning for multiple harmonics can be implemented via different digital coding sequences, thus removing the need for intricate phase-shift networks. This work paves the way for efficient harmonic control for applications in communications, radar, and related areas.
\end{abstract}

\section{Introduction}

Metasurfaces provide an unprecedented route to control light propagation and engineer light-matter interaction using an array of resonators with carefully designed geometries. With the introduction of an abrupt phase discontinuity along the interface, the electromagnetic (EM) properties of incident light can be tailored in a controlled manner as governed by the generalized Snell's law, resulting in full control of the propagation direction, polarization, and wavefront shape over subwavelength distances. These distinctive features have driven a rich variety of new technologies such as holographic imaging, perfect absorption and nonreciprocal transmission ${ }^{1-11}$.

\footnotetext{
Correspondence: Qiang Cheng (qiangcheng@seu.edu.cn) or

Tie Jun Cui (tjcui@seu.edu.cn)

${ }^{1}$ State Key Laboratory of Millimeter Waves, Southeast University, 210096

Nanjing, China

${ }^{2}$ Synergetic Innovation Center of Wireless Communication Technology,

Southeast University, 210096 Nanjing, China
}

With the rapid advent of various tuning technologies, a number of tunable metasurfaces have been shown to provide in situ dynamic control of electromagnetic waves, which opens a new route for a variety of applications such as beam shaping, active focusing and biosensing ${ }^{12-18}$.

However, one challenging task for the construction of a tunable metasurface involves the design of an intricate feeding network that provides various excitation intensities and phases to each element with high precision. In this regard, an alternative method has been explored, namely, coding the metasurface and digital metasurface, which shapes the phase front of the electromagnetic waves using binary meta-atoms with carefully designed geometries ${ }^{19-24}$. Such a configuration is extremely helpful to simplify the feeding network since only the discrete ON or OFF states for the modulation are required to manipulate the radiation or scattering properties of electromagnetic waves ${ }^{25-29}$.

\section{(c) The Author(s) 2018}

(c) (i) Open Access This article is licensed under a Creative Commons Attribution 4.0 International License, which permits use, sharing, adaptation, distribution and reproduction cc) in any medium or format, as long as you give appropriate credit to the original author(s) and the source, provide a link to the Creative Commons license, and indicate if changes were made. The images or other third party material in this article are included in the article's Creative Commons license, unless indicated otherwise in a credit line to the material. If material is not included in the article's Creative Commons license and your intended use is not permitted by statutory regulation or exceeds the permitted use, you will need to obtain permission directly from the copyright holder. To view a copy of this license, visit http://creativecommons.org/licenses/by/4.0/. 


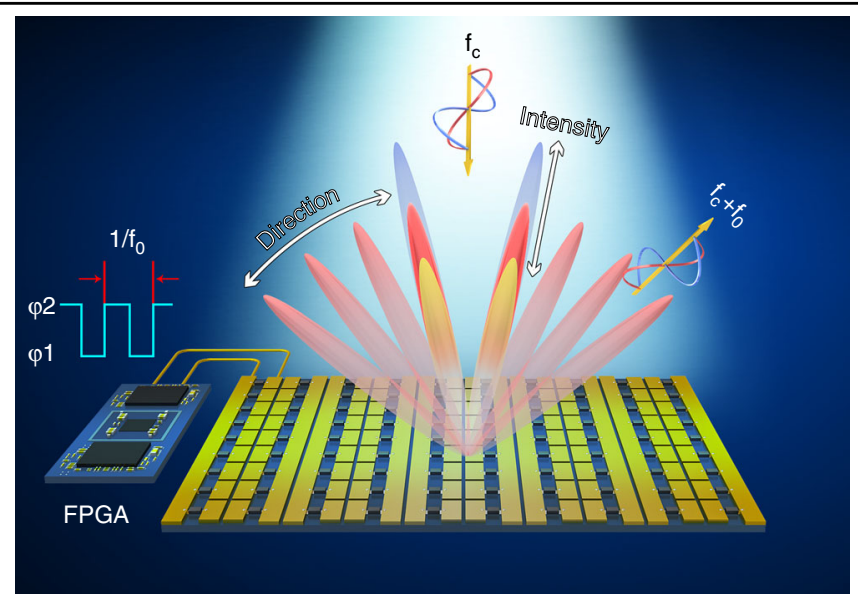

Fig. 1 Schematic diagram of the time-domain digital coding metasurface, which is able to generate nonlinear harmonics under excitation by a monochromatic wave as well as control their direction and magnitude

In addition to engineering the spectral response with a space-gradient metasurface, the time discontinuities of the element phase provide an additional degree of freedom to control the normal momentum component at the interface of the time-varying metasurface, leading to a break in Lorentz reciprocity during the light-matter interactions without the use of nonlinear materials ${ }^{30-39}$. Due to the rapid growth of communication technologies, the timemodulated antenna was developed to radiate harmonics with tight control of wave behavior so that information could be delivered through multichannels ${ }^{40-44}$. However, such an antenna lacks the ability to modulate incoming waves with efficient harmonic manipulation.

Here, we report on a scheme to achieve individual control of the amplitude and phase for harmonics via a reflective time-domain digital coding metasurface. As the reflection phase of the meta-atom is periodically switched between two states, a series of harmonics emerges, with their intensities determined by the phase difference within the switching function. Meanwhile, extra time delays are imposed on the modulation signal of the meta-atoms to create a metasurface with nonuniform harmonic phase distributions, thereby enabling wavefront reshaping for high-order harmonics. When discrete phase states of the meta-atoms are encoded into the binary sequences, control of multiple harmonics including the beam directions and intensities is realized using elaborate coding strategies. All measured signals agree closely with the analytical predictions, with these unique features likely to have key roles in future radar and communication systems.

\section{Results}

\section{Design of the metasurface}

We designed a reflective time-domain digital coding metasurface (Fig. 1) with each unit loaded with varactor diodes. The phase response of the meta-atom can be accurately tailored within a wide phase range $\left(\sim 270^{\circ}\right)$ by tuning the biasing voltage of the diode. This can be easily implemented when the DC feed of the element is connected to the peripheral circuit responsible for the generation of the desired control signal level, as will be discussed later. A three-dimensional schematic view of the meta-atom is shown in Fig. 2a. The side and central rectangular patches of the proposed element are bridged by four varactor diodes in total. A slotted copper layer lies at the bottom of the element to act as the DC feeding network, where a biasing voltage is applied to the diode via holes. A more detailed element description is provided in Fig. $2 \mathrm{~b}$, where $P_{\mathrm{x}}=37 \mathrm{~mm}, P_{\mathrm{y}}=33 \mathrm{~mm}, H=4 \mathrm{~mm}$, $M=16 \mathrm{~mm}, N=8.45 \mathrm{~mm}, L=7.45 \mathrm{~mm}, g=0.5 \mathrm{~mm}, d=$ $2.1 \mathrm{~mm}, t=4.6 \mathrm{~mm}, \Phi$ (diameter of the hole) $=1 \mathrm{~mm}$, and $s=0.3 \mathrm{~mm}$. The top and bottom layers are spaced by a substrate (F4B) with a dielectric constant and loss tangent of 2.65 and 0.001 , respectively. To prohibit wave transmission through the slots of the feeding layer, an extra copper layer is placed at the bottom of the metasurface, eliminating the possibility of energy leakage. In general, the varactor diode (SMV-2019, Skyworks, Inc.) can be regarded as an RLC model at the operation frequency with the equivalent circuit parameters listed in ref. ${ }^{45}$. To grasp a complete picture of the reflection spectra for the meta-atom, a full wave simulation was performed using a commercial electromagnetic solver (CST Microwave Studio 2016) under different biasing voltages. The reflection minima was observed to redshift (Fig. 2c) as the biasing voltage was changed from 19 to 0 V. Good phase linearity and a large phase range near 3.7 $\mathrm{GHz}$ was observed, as shown in Fig. 2d. This enables beam steering based on the relative phase shift of adjacent elements similar to the principle of the phase array antenna. The layout of the metasurface consists of $7 \times 8$ elements (Fig. 2). For simplicity, all the varactor diodes in 

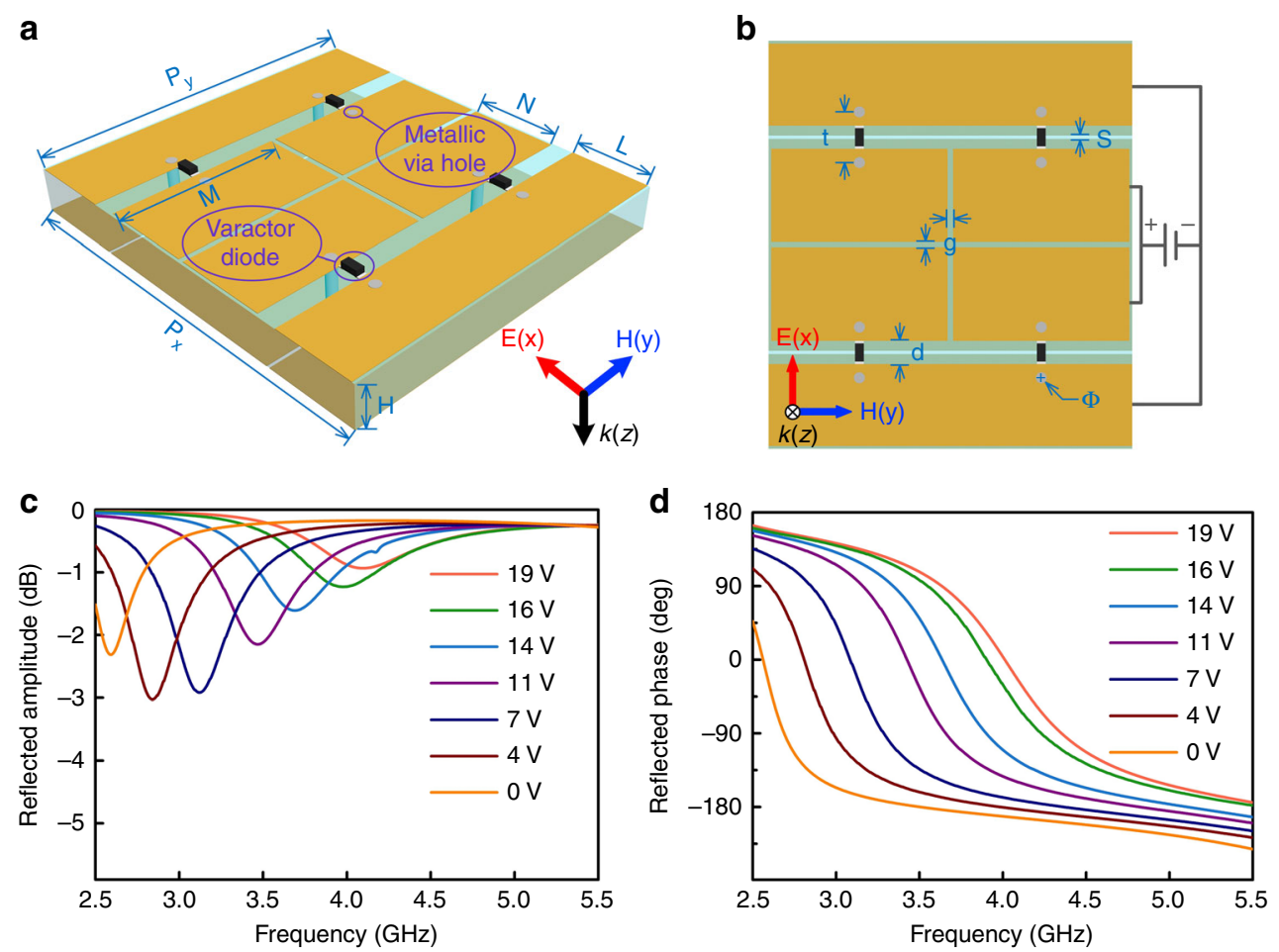

Fig. 2 Illustration of the time-domain digital coding meta-atom. $\mathbf{a}$, b Perspective and front views of the time-domain digital coding meta-atom. c, d The simulated reflection amplitude (c) and phase (d) spectra under different bias voltages. The dimensions of the meta-atom are $P_{\mathrm{x}}=37 \mathrm{~mm}$, $P_{y}=33 \mathrm{~mm}, H=4 \mathrm{~mm}, M=16 \mathrm{~mm}, N=8.45 \mathrm{~mm}, L=7.45 \mathrm{~mm}, g=0.5 \mathrm{~mm}, d=2.1 \mathrm{~mm}, t=4.6 \mathrm{~mm}, \Phi=1 \mathrm{~mm}, s=0.3 \mathrm{~mm}$

Table 1 The mapping relationship between the reflection phase of the meta-atom and biasing voltage at $3.7 \mathrm{GHz}$ in the simulation

\begin{tabular}{lllllllll}
\hline Voltage (V) & 0 & 3 & 6 & 9 & 12 & 15 & 18 & 21 \\
Phase (Deg.) & 0 & 10 & 30 & 90 & 180 & 210 & 250 & 270 \\
\hline
\end{tabular}

each column share the same biasing voltage. Targeting the discrete phases represented by the codes in our design, a series of biasing voltages, i.e., $0,3,6,9,12,15,18$, and 21 $\mathrm{V}$, were adopted to meet the phase demand in the experiment. The mapping relationship between the reflection phase and the biasing voltage for the meta-atom in the simulation is presented in Table 1, which provides a basis for the DC level on the diodes for each element in the following experiment.

\section{Experimental results}

During the experiment, two horn antennae were used to illuminate the sample and receive the reflected signal, respectively. Both the microwave signal generator and the spectrum analyzer were utilized to monitor the nonlinear properties of the metasurface over a broad spectrum when connected to the horn antennae separately through phase stable cables. The sample was fabricated using standard
PCB technology, with all components described in Fig. 3a and b. A FPGA controller, a digital-analog conversion (DAC) module and an analog amplifier module were employed to ensure a high output swing in accordance with the large range of regulation voltages used for the selected varactors. The operation frequency $f_{0}$ was 3.7 $\mathrm{GHz}$ and the control circuit was programmed to generate square wave control signals with different voltages, periods and time delays.

Prior to measurement of the scattering patterns of the harmonics, it was important to observe the nonlinear generation capability of the sample through experiments (see theory in Materials and methods). Using Table 1, which relates the biasing voltage to the reflection phase, it is easy to identify the combination of biasing voltages $V_{1} /$ $V_{2}$ corresponding to the required phases $\phi_{1}$ and $\phi_{2}$ in Eq. 4. During the experiment, we used a spectrum analyzer to directly measure the harmonic intensities. The harmonic phases were obtained through Fast Fourier Transformation of the echo signal from the metasurface, which was recorded by a software-defined radio reconfigurable device (USRP-2943, National Instruments Corp.). Figure 4 illustrates the relationship between the measured harmonic intensities/phases and the biasing voltage $V_{1} / V_{2}$ as well as the modulation period $T$ when all the varactor diodes of the metasurface are biased with the same control signal. 

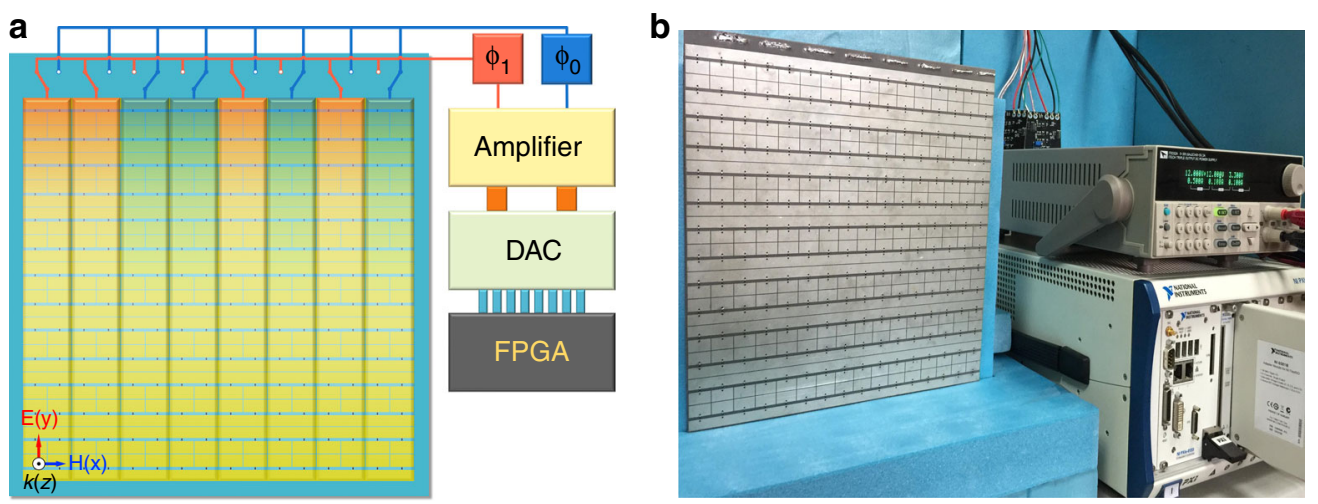

Fig. 3 Schematic and photograph of the time-domain digital coding metasurface. a Systematic description of the time-domain digital coding metasurface. $\mathbf{b}$ Photograph of the fabricated sample (on the left) in the experiment. The control system is presented in the right of $\mathbf{b}$, consisting of a DC power source, FPGA platform (NI Corp.), DAC module and an external amplifier module

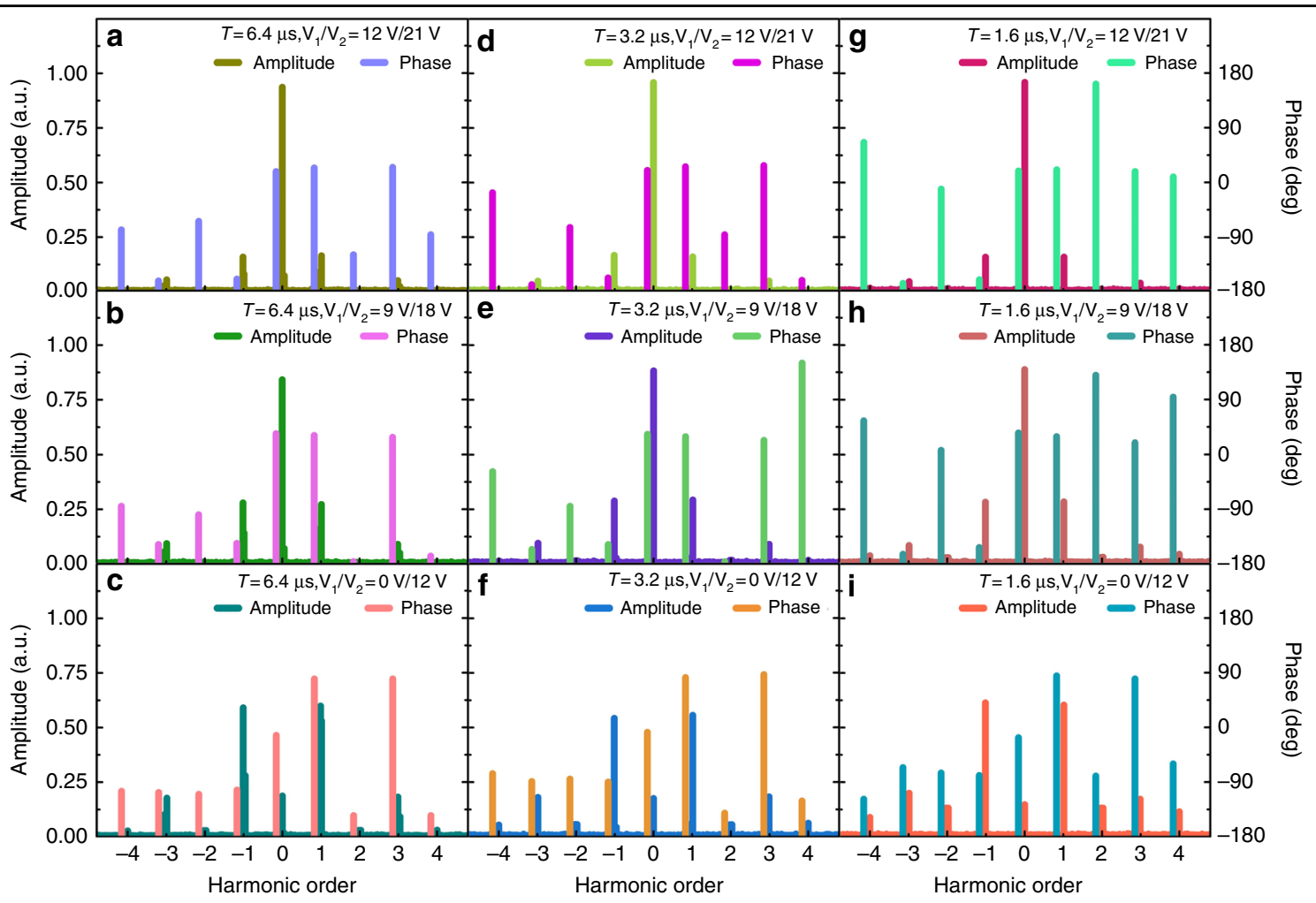

Fig. 4 The measured harmonic intensities/phases distribution of the time-domain digital coding metasurface at $3.7 \mathrm{GHz}$, where different biasing voltages $V_{\mathbf{1}} / V_{\mathbf{2}}$ and modulation periods $T$ are considered. The same modulation period $T=6.4 \mu \mathrm{s}(\mathbf{a}-\mathbf{c}), T=3.2 \mu \mathrm{s}(\mathbf{d}-\mathbf{f})$ and $T=1.6 \mu \mathrm{s}$ (g-i) are applied in each column, corresponding to a frequency interval of $156.25 \mathrm{kHz}, 312.5 \mathrm{kHz}$ and $625 \mathrm{kHz}$, while the same biasing voltage $V_{1} / V_{2}=$ $12 \mathrm{~V} / 21 \mathrm{~V}(\mathbf{a}-\mathbf{g}), V_{1} / V_{2}=9 \mathrm{~V} / 18 \mathrm{~V}(\mathbf{b}-\mathbf{h}), V_{1} / V_{2}=0 \mathrm{~V} / 12 \mathrm{~V}(\mathbf{c}-\mathbf{i})$ is adopted in each row, respectively

Although the measured results in Fig. 4 indicate that the mapping relationship between the biasing voltage and reflection phase is not entirely consistent with the designs in Table 1, the nonlinear phenomenon still occurs. Consistent with theoretical predictions, the measured results clearly reproduce the trend of growing harmonics as $\phi_{1}-\phi_{2}$ approaches $\pi$. In contrast, the intensity of the synchronous component experiences a significant drop as most of the reflection energy is shifted into the harmonic channels, which is expected to be totally eradicated in the case of opposite phases for $\phi_{1}$ and $\phi_{2}$ from Eq. (8). Such a discrepancy may be ascribed to phase deviations of the meta-atoms from their ideal values under different biasing voltages, largely stemming from the parasitic parameters 


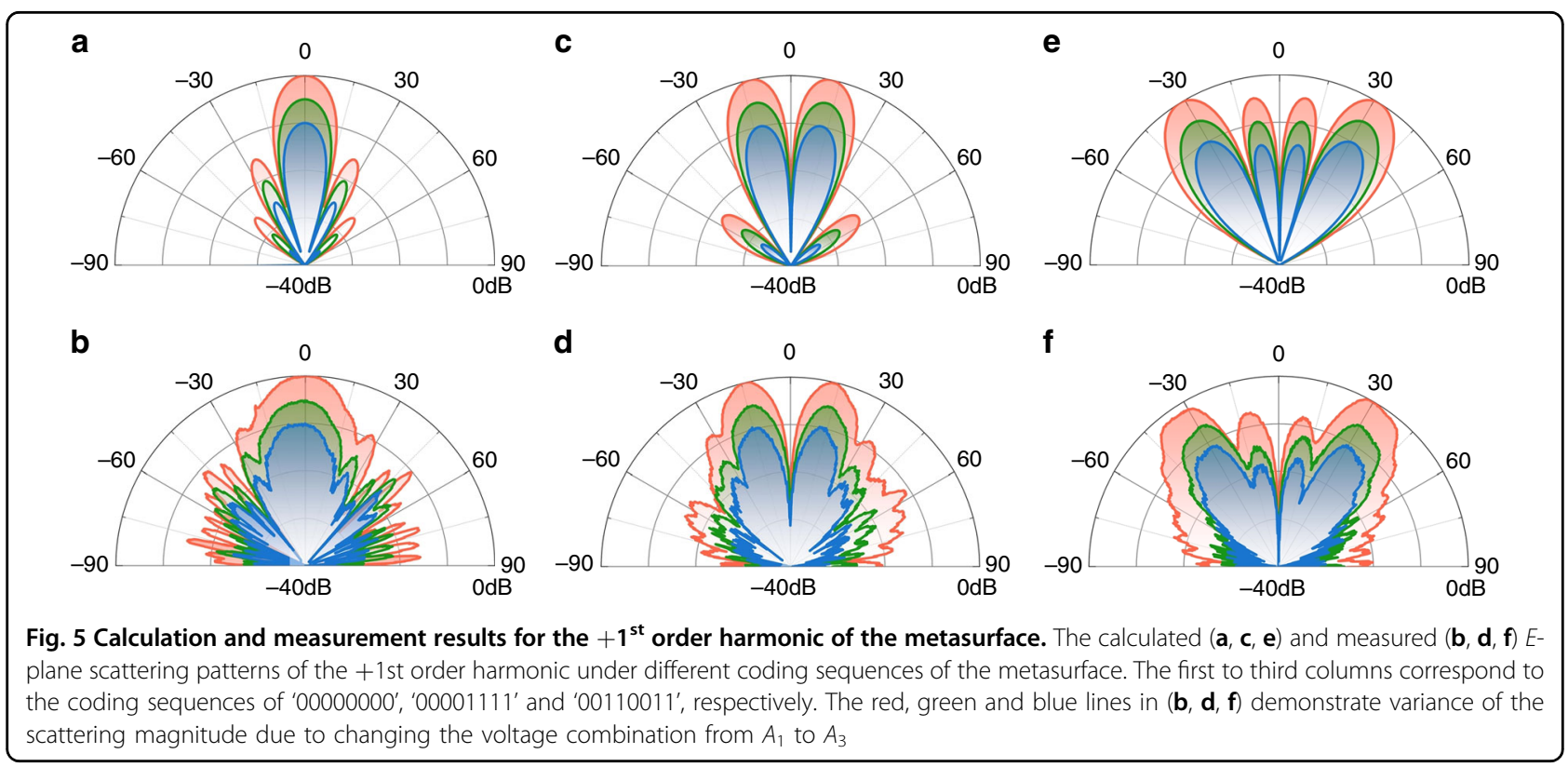

of the varactor diode, the fabrication tolerance and distortion of the control signal. The last issue is caused by the small switching time of the biasing signal that is beyond the bandwidth of the control circuit, which in turn damages the edges of the square waveform.

Figure 4 reveals that the modulation period has nearly no influence on the harmonic intensity but plays a vital role in determining the positions of the spectral lines. With a decrease of the modulation period from 6.4 to $1.6 \mu \mathrm{s}$, the observed frequency gap between adjacent harmonics is increased by up to $625 \mathrm{kHz}$ in the experiment, proportional to $1 / \mathrm{T}$. In addition to controlling the spectral position of the first harmonic, the possibility of amplitude modulation with the metasurface is shown in Fig. 4. For example, the amplitude of the $+1^{\text {st }}$ order harmonic is closely associated with the biasing voltage combination $V_{1}$ and $V_{2}$ from each column in Fig. 4. Therefore, the task of amplitude modulation can be easily implemented by tuning the external biasing voltages.

The designed metasurface offers a wide dynamic range of up to $25 \mathrm{~dB}$ for the $+1^{\text {st }}$ order harmonic amplitude with respect to different voltage combinations. This is ideally suited for accurate tuning of the harmonic intensity in practice. For better demonstration in the following experiment, three sets of parameters with $0 \mathrm{~V} / 12 \mathrm{~V}\left(A_{1}\right)$, $9 \mathrm{~V} / 18 \mathrm{~V}\left(A_{2}\right)$, and $12 \mathrm{~V} / 21 \mathrm{~V}\left(A_{3}\right)$ were picked up manually to meet the requirement for amplitude attenuation by 0,5 , and $10 \mathrm{~dB}$ for the $+1^{\text {st }}$ order harmonic when reflected from the sample.

We then experimentally investigated the modification of the scattering pattern of the $+1^{\text {st }}$ order harmonic via the proposed metasurface. The modulation period was $T=6.4 \mu \mathrm{s}$, indicating that the $+1^{\text {st }}$ order harmonic operates at a frequency of $3.70015625 \mathrm{GHz}$. Following the derivation in Eq. (8), the control signal shift in time $t_{0}$ can lead to an extra phase lag $\omega_{0} t_{0}$ for the $+1^{\text {st }}$ order harmonic without altering its magnitude (see Materials and methods). Thus, the time delay $0(0 \mu \mathrm{s})$ and $T / 2(3.2 \mu \mathrm{s})$ can be employed respectively to represent the coded elements ' 0 ' and ' 1 ' with opposite phase. Consequently, the reflection phases of all the columns in the metasurface (Fig. 3a) can be described by the coding sequence of ' 0 ' and ' 1 ' bits. In case of the metasurface encoded by '00000000' with no phase difference for all the columns, a directive scattering beam was measured for the $+1^{\text {st }}$ order harmonic along the normal direction, as shown in Fig. 5b. The biasing voltages also yielded magnitude control for the scattering pattern without changing its profile. By varying the voltage combination from $A_{1}$ to $A_{3}$, a decay rate of 0 , 5 , and $10 \mathrm{~dB}$ was observed for the scattering magnitude from the red, green and blue lines in Fig. 5b. Further characterization of the scattering patterns for the codes '00001111' and '00110011' can be found in Fig. 5d-f, in which the main scattering lobe was beamed toward different angular directions via the interference from the columns, enabling a large range of magnitude adjustment via control of the biasing voltages similar to that in Fig. 5b. By comparing Fig. 5a-e and Fig. 5b-f, one can observe a good correspondence between the theoretically calculated and experimentally measured results. Such agreement suggests potential for the beam forming the $+1^{\text {st }}$ order harmonic via the time-domain digital coding metasurface with sufficient accuracy.

Finally, we explored the feasibility of multiharmonic control with the same strategy. As mentioned above, the time delay $t_{0}$ of the control signal introduced an 
additional phase shift $k \omega_{0} t_{0}$ to the harmonic of $k^{\text {th }}$ order. This interesting property hints toward a novel route for controlling the scattering patterns of multiple harmonics at the same time. Here, we aimed to simultaneously tailor the scattering features of $-3^{\text {rd }},-1^{\text {st }},+1^{\text {st }}$, and $+3^{\text {rd }}$ order harmonics in experiment. To achieve a more flexible control of the harmonics, the 3-bit meta-atoms with eight phase states were adopted by applying various time delays $t_{0}$ to the square wave functions. The codes ' 0 ', ' 1 ', ' 2 ', ' 3 ', ' 4 ', ' 5 ' ' 6 ', ' 7 ' were used to represent $0, \pi / 4, \pi / 2,3 \pi / 4, \pi$, $5 \pi / 4,3 \pi / 2,7 \pi / 4$ for the $+1^{\text {st }}$ order harmonic when $t_{0}$ equals $0(0 \mu \mathrm{s}), T / 8(0.8 \mu \mathrm{s}), T / 4(1.6 \mu \mathrm{s}), 3 T / 8(2.4 \mu \mathrm{s}), T /$ $2(3.2 \mu \mathrm{s}), 5 T / 8(4 \mu \mathrm{s}), 3 T / 4(4.8 \mu \mathrm{s}), 7 T / 8(5.6 \mu \mathrm{s})$. However, the coding implications led to a great difference with changing harmonic order, which corresponds to $(0$, $-\pi / 4,-\pi / 2,-3 \pi / 4,-\pi,-5 \pi / 4,-3 \pi / 2,-7 \pi / 4),(0,3 \pi / 4$, $3 \pi / 2,9 \pi / 4,3 \pi, 15 \pi / 4,9 \pi / 2,21 \pi / 4)$ and $(0,-3 \pi / 4,-3 \pi / 2$, $-9 \pi / 4,-3 \pi,-15 \pi / 4,-9 \pi / 2,-21 \pi / 4)$ for $-1^{\text {st }},+3^{\text {rd }}$, and $-3^{\text {rd }}$ order harmonics, respectively. This change means that these harmonics have different scattering orientations under the same coding sequences. The $-3^{\text {rd }},-1^{\text {st }}$, $+1^{\text {st }}$, and $+3^{\text {rd }}$ order harmonics were set to operate at $3.69953125,3.69984375,3.70015625$, and 3.70046875 $\mathrm{GHz}$, respectively. We investigated the waveform modulation phenomena over the aforementioned four harmonics when switching among the predesigned coding sequences. Figure 6 demonstrates the measured E-plane scattering patterns of the $-3^{\text {rd }}$ (purple line), $-1^{\text {st }}$ (blue line), $+1^{\text {st }}$ (red line) and $+3^{\text {rd }}$ (green line) order harmonics monitored by the spectrum analyzer, along with the corresponding simulation results. Five coding schemes were applied to the eight columns of the metasurface from left to right (Fig. 3), including '00000000', '00112334', '00111122', '33221100', and '76543210'. The calculated results (dashed lines) show how the variance of the coding sequence affects the scattering response of the four harmonics under normal illumination by plane waves at $3.7 \mathrm{GHz}$, based on the Fourier transformation between the surface currents and the far fields ${ }^{46,47}$. Due to the linear relationship between the phase shift and the harmonic order, it was possible to achieve a larger deflection angle for high-order harmonics. This can be confirmed by comparing the scattering patterns with the same coding sequence in each line of Fig. 6. Moreover, symmetric scattering patterns could be found for the harmonic pairs $\left(-1^{\text {st }},+1^{\text {st }}\right)$ and $\left(-3^{\text {rd }},+3^{\text {rd }}\right)$ (Fig. 6) since opposite phase distributions were obtained with the same coding sequence, which thus gave rise to reversed in-plane momentum added to that of the incident wave. The close agreement between the measured and calculated scattering angles suggests that the time-domain digital coding metasurface can serve as a good candidate to realize a spatial scan with multiple harmonics, e.g., one can simultaneously scan the angular region from $0^{\circ}$ to $90^{\circ}$ by the $+1^{\text {st }}$ order harmonic and the region from $-90^{\circ}$ to $0^{\circ}$ by the $-1^{\text {st }}$ order harmonic, and thus reduce the time cost for measurement by half.

\section{Discussion}

In this study, we created a time-domain digital coding metasurface to generate harmonics under the illumination of electromagnetic waves with a high conversion rate, which enables independent control of the harmonic amplitude and phase. By controlling the biasing voltages of the varactor diodes incorporated into the meta-atoms, it was possible to acquire a periodic time-variant reflection coefficient responsible for regulating the harmonic intensity. Meanwhile, the time delay of the switching functions for the meta-atoms can be carefully selected to modify the harmonic reflection phase, so that the metasurface can be employed to reshape the wavefront for multiple harmonics. Although our work was experimentally confirmed at microwave frequencies, it can be further extended into the $\mathrm{THz}$ and light regime in combination with advanced modulation technologies ${ }^{32,33}$. Furthermore, the transmission type metasurface can also be employed to realize harmonic manipulation when it is fed by a patch antenna array from behind.

\section{Materials and methods}

\section{The nonlinear theory for the time-domain digital coding} metasurface

We started by considering the reflection problem when EM waves are illuminated normally from the upper free space upon a reflective time-domain digital coding metasurface. The temporal expression of the reflected wave can be written as $E_{r}(t)=\Gamma(t) \cdot E_{i}(t)$, where $E_{r}(t), E_{i}(t)$ and $\Gamma(t)$ denote the reflected wave, incident wave, and reflection coefficient, respectively. Specifically, in the case of monochromic incidence at frequency $f_{c}$ where $E_{i}(t)=e^{-j \omega_{c} t}$, the reflected wave in the frequency domain reads

$$
E_{r}(\omega)=\Gamma(\omega) *\left[\delta\left(\omega-\omega_{c}\right)\right]=\Gamma\left(\omega-\omega_{c}\right)
$$

where $\omega_{c}$ the is angular frequency and $\delta\left(\omega-\omega_{c}\right)$ represents the Dirac delta function at $\omega=\omega_{c}$. From Eq. 1, it is straightforward to find that for a time-invariant metasurface with constant $\Gamma(t)$, the reflected signal only contains information for the carrier frequency $\omega_{c}$. However, if the reflection coefficient $\Gamma(t)$ turns out to be a periodic signal, it can be expressed as a linear combination of harmonically related complex exponentials

$$
\Gamma(t)=\sum_{k=-\infty}^{+\infty} a_{k} e^{j k \omega_{0} t}=\sum_{k=-\infty}^{+\infty} a_{k} e^{j k \frac{2 \pi}{T} t}
$$

with the corresponding spectral expression for the 


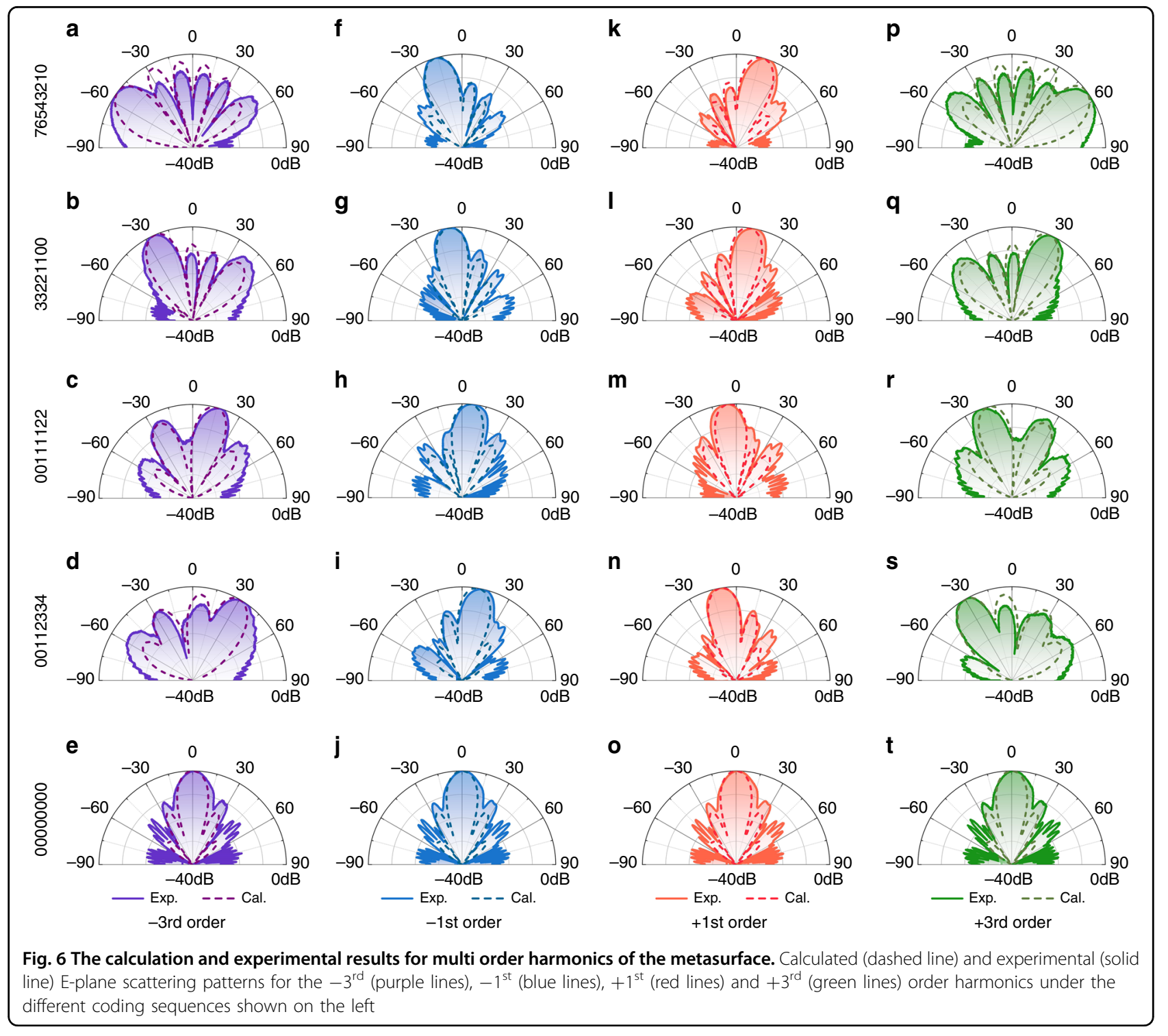

reflected wave given as follows

$$
E_{r}(\omega)=2 \pi \sum_{k=-\infty}^{+\infty} a_{k} E_{i}\left(\omega-k \omega_{0}\right)
$$

in which $\omega=2 \pi / T$ represents the angular frequency determined by the function period $T$ and $a_{k}$ is the coefficient of the $k^{\text {th }}$ harmonic component. Due to strong wave-matter interactions, a nonlinear phenomenon occurs as characterized by the emergence of numerous harmonics beside the fundamental components. Here, we focus on a specific scenario of a lossless metasurface with a reflection phase response described by the periodic square wave

$$
\Gamma(t)=A e^{j\left\{\phi_{1}+\left(\phi_{2}-\phi_{1}\right) \sum_{n=-\infty}^{+\infty}\left[\varepsilon(t-n T)-\varepsilon\left(t-\frac{T}{2}-n T\right)\right]\right\}}
$$

where $A$ is the constant reflection amplitude, $\phi_{1}, \phi_{2}$ are the two phase states of the square wave and $\varepsilon(t-n T)$ represents the unit step function shifted by $n T$. From Eqs. (2)-(4), the harmonic coefficient is given by:

$$
a_{k}= \begin{cases}A \cos \frac{\phi_{2}-\phi_{1}}{2} e^{j \frac{\phi_{2}+\phi_{1}}{2}} & k=0 \\ \frac{2 A}{k \pi} \sin \frac{\phi_{2}-\phi_{1}}{2} e^{\phi_{2} \frac{\phi_{1}}{2}} & k= \pm 1, \pm 3, \pm 5 \cdots \\ 0 & k= \pm 2, \pm 4, \pm 6 \cdots\end{cases}
$$

The Fourier transform of Eq. (5) yields

$$
\begin{aligned}
E_{r}(\omega) & =2 \pi A \cos \frac{\phi_{2}-\phi_{1}}{2} e^{\frac{\phi_{2}+\phi_{1}}{2}} E_{i}(\omega) \\
& +\sum_{m=-\infty}^{+\infty} \frac{4 A}{2 m-1} \sin \frac{\phi_{2}-\phi_{1}}{2} e^{j \frac{\phi_{2}+\phi_{1}}{2}} E_{i}\left[\omega-(2 m-1) \omega_{0}\right]
\end{aligned}
$$




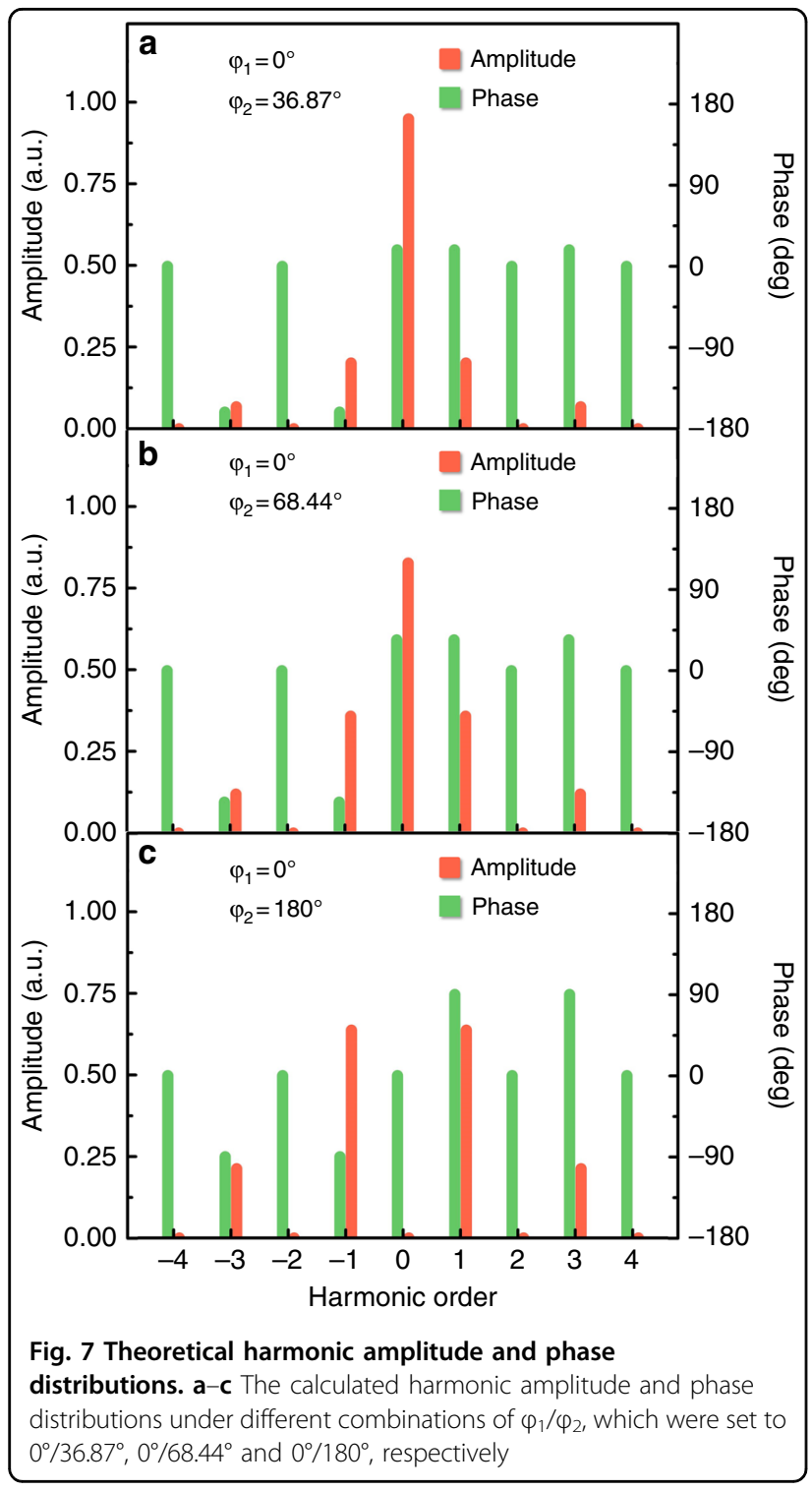

Only the synchronous component $(k=0)$ and odd harmonics are found to survive in the reflected waves, as can be understood in terms of the Fourier Transform of square waves. The envelope of the reflection spectra is highly associated with the phase pair $\left(\phi_{1}, \phi_{2}\right)$, which in turn offers the opportunity to dynamically control the amplitude and phase distributions of high-order harmonics. To further illustrate this principle, the dependence of the reflection spectra on $\phi_{1}$ and $\phi_{2}$ is illustrated in Fig. 7 . Three sets of phase combinations are taken into account with $\phi_{1} / \phi_{2}=0^{\circ} / 36.87^{\circ}, 0^{\circ} / 68.44^{\circ}$ and $0^{\circ} / 180^{\circ}$, respectively. As the phase difference $\Delta \phi=\phi_{1}-\phi_{2}$ gradually approaches $180^{\circ}$, the intensity of the synchronous component tends to be significantly suppressed, as shown in Fig. 7a-c. This phenomenon is consistent with the mathematical predictions in Eq. 6, where the first term that represents the synchronous component is proportional to the cosine function of $\Delta \phi / 2$. Meanwhile, as a consequence, the harmonic energies are augmented, resulting in efficient frequency conversion. When the opposite phase requirement between $\phi_{1}$ and $\phi_{2}$ is satisfied, the synchronous components are totally cancelled under this circumstance, whereas the harmonics of the $\pm 1^{\text {st }}$ order reach the maximum amplitude with $a_{1}=0.6366$. The calculated data in Fig. 7 were used to determine the contribution of the harmonics to overall reflection, which is dominated by the components $k= \pm 1$, accounting for 81.05 percent of the overall reflection energy in Fig. 7c. Such a proportion is especially favored for mixing applications, leading to new opportunities for up and down conversion with satisfactory efficiency in free space.

\section{Independent control of the harmonic amplitudes and phases by the time-domain digital coding metasurface}

Until now, we have only considered the feasibility of harmonic generation through the time-domain digital coding metasurface. One drawback of the proposed scheme is the strong correlation between the harmonic amplitude and phase, thereby greatly hindering independent control of both parameters in practice. A possible recipe to overcome such limitation lies in the introduction of an additional time delay $t_{0}$ to the time-varying reflection coefficient. Following the time shift property of a Fourier transform with $\Gamma\left(t-t_{0}\right)=\mathcal{F}^{-1}\left[e^{-j \omega t_{0}} \Gamma(j \omega)\right]$, the time delay leads to an additional phase shift $e^{-j k \omega_{0} t_{0}}$ for the $k^{\text {th }}$ order harmonic, while maintaining an unchanged amplitude. After a more formal derivation, we obtain the spectral expression for the reflected wave in this case

$$
\begin{array}{r}
E_{r}(\omega)=2 \pi A \cos \frac{\phi_{2}-\phi_{1}}{2} e^{\frac{j \phi_{2}+\phi_{1}}{2}} E_{i}(\omega) \\
+\sum_{m=-\infty}^{+\infty} \frac{4 A}{2 m-1} \sin \frac{\phi_{2}-\phi_{1}}{2} e^{j\left[\frac{\phi_{2}+\phi_{1}}{2}-(2 m-1) \omega_{0} t_{0}\right]} \\
E_{i}\left[\omega-(2 m-1) \omega_{0}\right]
\end{array}
$$

Specifically, the amplitude and phase of the $k^{\text {th }}$ order harmonic are rewritten as

$$
\begin{aligned}
& E_{r}\left(k \omega_{0}+\omega_{c}\right)=|A|\langle\Phi \\
& = \begin{cases}\left|A \cos \frac{\phi_{2}-\phi_{1}}{2}\right| e^{j\left\{\frac{\phi_{2}+\phi_{1}}{2}+\pi\left[\varepsilon\left(\frac{\phi_{2}-\phi_{1}-\pi}{2}\right)+\varepsilon\left(\frac{\phi_{1}-\phi_{2}-\pi}{2}\right)\right]\right\}} & k=0 \\
\left|\frac{2 A}{k \pi} \sin \frac{\phi_{2}-\phi_{1}}{2}\right| e^{j\left\{\frac{\phi_{2}+\phi_{1}}{2}-\pi\left[\varepsilon\left(\phi_{2}-\phi_{1}\right)+\varepsilon(k)\right]-k \omega_{0} t_{0}\right\}} & k= \pm 1, \pm 3, \pm 5 \cdots \\
0 & k= \pm 2, \pm 4, \pm 6 \cdots\end{cases}
\end{aligned}
$$

The extra phase shift provides new degrees of freedom to control the propagation features of high-order harmonics by breaking the constraint between their amplitude and phase, making it possible to realize independent regulation of both parameters, which is hard to achieve using conventional methods. In fact, Eq. (8) offers a promising route to tailor the response of reflected 
Table 2 Measured and theoretical conversion efficiency when $V_{1} / V_{2}\left(\varphi_{1} / \varphi_{2}\right)=0 \mathrm{~V} / 12 \mathrm{~V}\left(0^{\circ} / 180^{\circ}\right)$ with $T=6.4 \mu \mathrm{s}$

\begin{tabular}{|c|c|c|c|}
\hline $\begin{array}{l}\text { Harmonic } \\
\text { order }\end{array}$ & $\begin{array}{l}\text { Measured } V_{1} / \\
V_{2}=0 \mathrm{~V} / 12 \mathrm{~V}\end{array}$ & $\begin{array}{l}\text { Theoretical } \varphi_{1} / \\
\varphi_{2}=0^{\circ} / 180^{\circ}\end{array}$ & Error \\
\hline$-4^{\text {th }}$ & $0.08 \%$ & $0 \%$ & $0.08 \%$ \\
\hline$-3^{r d}$ & $3.14 \%$ & $4.50 \%$ & $-1.36 \%$ \\
\hline$-2^{\text {nd }}$ & $0.08 \%$ & $0 \%$ & $0.08 \%$ \\
\hline$-1^{\mathrm{st}}$ & $34.93 \%$ & $40.53 \%$ & $-5.60 \%$ \\
\hline $0^{\text {th }}$ & $3.49 \%$ & $0 \%$ & $3.49 \%$ \\
\hline$+1^{\mathrm{st}}$ & $35.87 \%$ & $40.53 \%$ & $-4.66 \%$ \\
\hline$+2^{\text {nd }}$ & $0.09 \%$ & $0 \%$ & $0.09 \%$ \\
\hline$+3^{r d}$ & $3.31 \%$ & $4.50 \%$ & $-1.19 \%$ \\
\hline$+4^{\text {th }}$ & $0.10 \%$ & $0 \%$ & $0.10 \%$ \\
\hline
\end{tabular}

harmonics via the combination of three parameters $\phi_{1}, \phi_{2}$, and $t_{0}$, consequently pushing the intriguing functionalities accomplished by traditional metasurfaces into the nonlinear regime.

The concept of a digital coding metasurface arises from a simple analogy with digital circuits, where a finite number of element types are employed to manipulate electromagnetic waves. For a 1-bit metasurface, only two kinds of elements (named as ' 0 ' and ' 1 ' element) with opposite phase responses are utilized to constitute the entire structure. By alternating the spatial alignment of both elements, the metasurface demonstrates a powerful capability to reach a broad range of functionalities such as anomalous beam reflections and random diffusion. Such ideas can be easily extended to design of the metasurface in the time domain. For example, given the phase states $\phi_{1}, \phi_{2}$ in Eq. (8), it is easy to construct a ' 0 ' and ' 1 ' element by inserting a time delay $t_{0}$ for the $k^{\text {th }}$ order harmonic. Here, we choose $t_{0}=0$ and $T / 2$ respectively, in which $T$ is the period of the square function in Eq. (4). As a quantitative illustration of nonlinear control, we considered a scenario where a plane wave is illuminated normally at $f_{\mathrm{c}}=3.7 \mathrm{GHz}$ upon the 1-bit time-domain digital coding metasurface. In this case, we use eight columns of metaatoms in total with a unit period $\mathrm{P}=33 \mathrm{~mm}$. The repeating frequency of the square wave was $f_{0}=156.25$ $\mathrm{kHz}$. Figure $5 \mathrm{a}, \mathrm{c}$, e shows the calculated E-plane scattering pattern for the $+1^{\text {st }}$ order harmonic under different coding sequences '0000...', '00001111...', and '00110011...'. From the antenna theory, the scattering pattern is shown to hold as a Fourier transform pair with the phase distributions within the metasurface. Therefore, the scattering pattern can be directly inferred from the coding sequences of the binary elements through mathematical calculations. The backward scattering beam is split into two symmetric beams directed along either side,
Table 3 Measured and theoretical conversion efficiency when $V_{1} / V_{2}\left(\varphi_{1} / \varphi_{2}\right)=9 \mathrm{~V} / 18 \mathrm{~V}\left(0^{\circ} / 68.44^{\circ}\right)$ with $T=6.4 \mu \mathrm{s}$

\begin{tabular}{llll}
\hline $\begin{array}{l}\text { Harmonic } \\
\text { order }\end{array}$ & $\begin{array}{l}\text { Measured } \boldsymbol{V}_{\mathbf{1}} / \\
\boldsymbol{V}_{\mathbf{2}}=\mathbf{9} \mathbf{V} / \mathbf{1 8} \mathbf{V}\end{array}$ & $\begin{array}{l}\text { Theoretical } \boldsymbol{\varphi}_{\mathbf{1}} / \\
\boldsymbol{\varphi}_{\mathbf{2}}=\mathbf{0}^{\circ} \mathbf{6 8 . 4 \mathbf { 4 4 } ^ { \circ }}\end{array}$ & Error \\
\hline$-4^{\text {th }}$ & $0.01 \%$ & $0 \%$ & $0.01 \%$ \\
$-3^{\text {rd }}$ & $0.87 \%$ & $1.42 \%$ & $-0.55 \%$ \\
$-2^{\text {nd }}$ & $0.01 \%$ & $0 \%$ & $0.01 \%$ \\
$-1^{\text {st }}$ & $7.83 \%$ & $12.82 \%$ & $-4.99 \%$ \\
$0^{\text {th }}$ & $70.90 \%$ & $68.38 \%$ & $2.52 \%$ \\
$+1^{\text {st }}$ & $7.43 \%$ & $12.82 \%$ & $-5.39 \%$ \\
$+2^{\text {nd }}$ & $0.01 \%$ & $0 \%$ & $0.01 \%$ \\
$+3^{\text {rd }}$ & $0.81 \%$ & $1.42 \%$ & $-0.61 \%$ \\
$+4^{\text {th }}$ & $0.01 \%$ & $0 \%$ & $0.01 \%$ \\
\hline
\end{tabular}

with the tilting angle gradually increased, as shown in Fig. 5a, c, e. Such scattering behavior is closely associated with the introduction of the parallel wave-vector that is imposed onto the reflected wave with the change of spatial arrangement for the ' 0 ' and ' 1 ' elements.

In addition, the red, blue and green lines in Fig. 5a, c, e demonstrate the dependence of the scattering magnitude of the $+1^{\text {st }}$ order harmonic under different phase combinations of $\phi_{1} / \phi_{2}$ as $0^{\circ} / 180^{\circ}, 0^{\circ} / 68.44^{\circ}$ and $0^{\circ} / 36.87^{\circ}$, respectively. The scattering pattern undergoes a uniform attenuation in its amplitude by 0,5 , and $10 \mathrm{~dB}$ with the change in phase pair.

One important concern related to the metasurface is the conversion efficiency from the fundamental harmonic to high-order harmonics. To address this issue, we compare the measured and theoretical conversion efficiency of the $-4^{\text {th }}$ to $+4^{\text {th }}$ order harmonics in Tables 2 and 3, with $V_{1} /$ $V_{2}\left(\phi_{1} / \phi_{2}\right)=\mathrm{V} / 12 \mathrm{~V}\left(0^{\circ} / 180^{\circ}\right), T=6.4 \mu$ s and $V_{1} / V_{2}\left(\phi_{1} / \phi_{2}\right)$ $=\mathrm{V} / 18 \mathrm{~V}\left(0^{\circ} / 68.44^{\circ}\right), T=6.4 \mu \mathrm{s}$, respectively. The efficiency is obtained from the energy ratio between the harmonic and incident wave. From Table 2, when $\phi_{1}$ and $\phi_{2}$ are out of phase, the measured fundamental wave occupies $3.49 \%$ of the total energy, indicating that $96.51 \%$ of the incident power is converted to high-order harmonics. The majority of the energy is assigned to $\pm 1^{\text {st }}$ harmonics, as expected. In general, the theoretical and measured results for each harmonic are in good accordance. In the measurement, when $\phi_{1}-\phi_{2}=68.44^{\circ}$, only $30 \%$ of the incident energy is converted into high-order harmonics, consistent with the theoretical prediction in Table 3.

\section{Acknowledgements}

This work is supported by the National Key Research and Development Program of China under Grant Nos. 2017YFA0700201, 2017YFA0700202, 2017YFA0700203, the National Science Foundation of China (61631007, 
$61571117,61138001,61371035,61722106,61731010,11227904)$, and the 111 Project (111-2-05).

\section{Author contributions}

J.Y.D., J.Z., Q.C., and T.J.C. contributed equally to this work. J.Y.D. carried out the analytical modeling, numerical simulations, sample fabrication, and measurements together with J.Z. Q.C. and T.J.C. conceived the idea, suggested the designs, planned, coordinated, and supervised the work. All authors discussed the theoretical and numerical aspects and interpreted the results. All authors contributed to the preparation and writing of the manuscript.

\section{Conflict of interest}

The authors declare that they have no conflict of interest.

Received: 16 May 2018 Revised: 23 October 2018 Accepted: 3 November 2018

Published online: 21 November 2018

\section{References}

1. Ni, X. J., Kildishev, A. V. \& Shalaev, V. M. Metasurface holograms for visible light. Nat. Commun. 4, 2807 (2013).

2. Huang, L. L. et al. Three-dimensional optical holography using a plasmonic metasurface. Nat. Commun. 4, 2808 (2013).

3. Liu, L. X. et al. Broadband metasurfaces with simultaneous control of phase and amplitude. Adv. Mater. 26, 5031-5036 (2014).

4. Zheng, G. X. et al. Metasurface holograms reaching $80 \%$ efficiency. Nat. Nanotechnol. 10, 308-312 (2015).

5. Landy, N. I., Sajuyigbe, S., Mock, J. J., Smith, D. R. \& Padilla, W. J. Perfect metamaterial absorber. Phys. Rev. Lett. 100, 207402 (2008).

6. Liu, N., Mesch, M., Weiss, T., Hentschel, M. \& Giessen, H. Infrared perfect absorber and its application as plasmonic sensor. Nano Lett. 10, 2342-2348 (2010).

7. Mei, J. et al. Dark acoustic metamaterials as super absorbers for low-frequency sound. Nat. Commun. 3, 756 (2012).

8. Watts, C. M., Liu, X. L. \& Padilla, W. J. Metamaterial electromagnetic wave absorbers. Adv. Mater. 24, OP98-OP120 (2012)

9. Akselrod, G. M. et al. Large-area metasurface perfect absorbers from visible to near-infrared. Adv. Mater. 27, 8028-8034 (2015).

10. Sounas, D. L., Caloz, C. \& Alù, A. Giant non-reciprocity at the subwavelength scale using angular momentum-biased metamaterials. Nat. Commun. 4, 2407 (2013).

11. Popa, B. I. \& Cummer, S. A. Non-reciprocal and highly nonlinear active acoustic metamaterials. Nat. Commun. 5, 3398 (2014).

12. Sievenpiper, D. F., Schaffner, J. H., Song, H. J., Loo, R. Y. \& Tangonan, H. Twodimensional beam steering using an electrically tunable impedance surface. IEEE Trans. Antennas Propag. 51, 2713-2722 (2003).

13. Yao, Y. et al. Electrically tunable metasurface perfect absorbers for ultrathin mid-infrared optical modulators. Nano Lett. 14, 6526-6532 (2014).

14. Dabidian, N. et al. Electrical switching of infrared light using graphene integration with plasmonic Fano resonant metasurfaces. ACS Photonics 2, 216-227 (2015).

15. Chen, K. et al. A reconfigurable active Huygens' metalens. Adv. Mater. 29, 1606422 (2017).

16. Chen, J. N. et al. Optical nano-imaging of gate-tunable graphene plasmons. Nature 487, 77-81 (2012).

17. Rodrigo, D. et al. Mid-infrared plasmonic biosensing with graphene. Science 349, 165-168 (2015).

18. Lee, J. et al. Ultrafast electrically tunable polaritonic metasurfaces. Adv. Opt. Mater. 2, 1057-1063 (2014).

19. Cui, T. J., Qi, M. Q., Wan, X., Zhao, J. \& Cheng, Q. Coding metamaterials, digital metamaterials and programmable metamaterials. Light Sci. Appl. 3, e218 (2014).
20. Della Giovampaola, C. \& Engheta, N. Digital metamaterials. Nat. Mater. 13 1115-1121 (2014).

21. Cui, T. J., Liu, S., \& Li, L. L. Information entropy of coding metasurface. Light Sci. Appl. 5, e16172 (2016).

22. Cui, T. J. Microwave metamaterials. Natl. Sci. Rev. 5, 134-136 (2018).

23. Cui, T. J., Liu, S. \& Zhang, L. Information metamaterials and metasurfaces. J. Mater. Chem. C 5, 3644-3668 (2017).

24. Cui, T. J. Microwave metamaterials - from passive to digital and programmable controls of electromagnetic waves. J. Opt. 19, 084004 (2017).

25. Debogovic, T. \& Perruisseau-Carrier, J. Low loss MEMS-reconfigurable 1-bit reflectarray cell with dual-linear polarization. IEEE Trans. Antennas Propag. 62 5055-5060 (2014)

26. Wan, X., Qi, M. Q., Chen, T. Y. \& Cui, T. J. Field-programmable beam reconfiguring based on digitally-controlled coding metasurface. Sci. Rep. 6, 20663 (2016).

27. Li, Y. B. et al. Transmission-type 2-bit programmable metasurface for singlesensor and single-frequency microwave imaging. Sci. Rep. 6, 23731 (2016).

28. Huang, C. et al. Dynamical beam manipulation based on 2-bit digitally-controlled coding metasurface. Sci. Rep. 7, 42302 (2017).

29. Li, L. L. et al. Electromagnetic reprogrammable coding-metasurface holograms. Nat. Commun. 8, 197 (2017)

30. Hadad, Y., Sounas, D. L. \& Alu, A. Space-time gradient metasurfaces. Phys. Rev. B 92, 100304 (2015)

31. Shaltout, A., Kildishev, A. \& Shalaev, V. Time-varying metasurfaces and Lorentz non-reciprocity. Opt. Mater. Exp. 5, 2459-2467 (2015).

32. Liu, Z. Z., Li, Z. Y. \& Aydin, K. Time-varying metasurfaces based on graphene microribbon arrays. ACS Photonics 3, 2035-2039 (2016).

33. Stewart, S. A., Smy, T. J. \& Gupta, S. Finite-difference time-domain modeling of space-time-modulated metasurfaces. IEEE Trans. Antennas Propag. 66, 281-292 (2017).

34. Li, G. X. et al. Continuous control of the nonlinearity phase for harmonic generations. Nat. Mater. 14, 607-612 (2015).

35. Rose, A., Huang, D. \& Smith, D. R. Controlling the second harmonic in a phasematched negative-index metamaterial. Phys. Rev. Lett. 107, 063902 (2011).

36. Lee, J. et al. Giant nonlinear response from plasmonic metasurfaces coupled to intersubband transitions. Nature 511, 65-69 (2014).

37. Klein, M. W., Enkrich, C., Wegener, M. \& Linden, S. Second-harmonic generation from magnetic metamaterials. Science 313, 502-504 (2006).

38. Lapine, M., Shadrivov, I. V., Powell, D. A. \& Kivshar, Y. S. Magnetoelastic metamaterials. Nat. Mater. 11, 30-33 (2011).

39. Zhao, J. et al. Controlling spectral energies of all harmonics in programmable way using time-domain digital coding metasurface. Preprint at https:/arxiv. org/abs/1806.04414. Accessed June 2018.

40. Rocca, P., Zhu, Q. J., Bekele, E. T., Yang, S. W. \& Massa, A. 4-D arrays as enabling technology for cognitive radio systems. IEEE Trans. Antennas Propag. 62, 1102-1116 (2014).

41. Secmen M., Demir S., Hizal A., \& Eker T. Frequency diverse array antenna with periodic time modulated pattern in range and angle. In Proc. of 2007 IEEE Radar Conference 427-430 (IEEE, Boston, MA, USA, 2007).

42. Poli, L., Rocca, P., Manica, L. \& Massa, A. Handling sideband radiations in timemodulated arrays through particle swarm optimization. IEEE Trans. Antennas Propag. 58, 1408-1411 (2010).

43. Poli, L., Rocca, P., Oliveri, G. \& Massa, A. Harmonic beamforming in timemodulated linear arrays. IEEE Trans. Antennas Propag. 59, 2538-2545 (2011).

44. Poli, L., Rocca, P., Manica, L. \& Massa, A. Pattern synthesis in time-modulated linear arrays through pulse shifting. IET Microw. Antennas Propag. 4, 1157-1164 (2010).

45. Zhao, J. et al. A tunable metamaterial absorber using varactor diodes. New J. Phys. 15, 043049 (2013).

46. Liu, S. et al. Convolution operations on coding metasurface to reach flexible and continuous controls of terahertz beams. Adv. Sci. 3, 1600156 (2016).

47. Wang, D., Liu, Z. G., Zhao, J., Cheng, Q. \& Cui, T. J. Accurate design of low backscattering metasurface using iterative fourier transform algorithm. Sci. Rep. 7. 11346 (2017) 